\title{
Vitrectomy for idiopathic epiretinal membranes causing macular pucker
}

\author{
SERGE DE BUSTROS,' JOHN T THOMPSON,' RONALD G MICHELS, \\ THOMAS A RICE, ${ }^{2}$ AND BERT M GLASER'
}

From the 'Wilmer Ophthalmological Institute of the Johns Hopkins University, Baltimore, and the 'Department of Ophthalmology, Case Western Reserve University, School of Medicine, Cleveland, USA

SUMMARY We used vitreous surgery to remove idiopathic epiretinal membranes from the macular area in 70 consecutive cases. The abnormal tissue was successfully removed in each case. Vision improved postoperatively in 61 eyes $(87 \%)$, remained unchanged in six eyes $(9 \%)$, and worsened in three eyes $(4 \%)$. However, at the time of final examination vision was improved in only 47 eyes $(67 \%)$, primarily because of the occurrence or progression of nuclear sclerosis, which occurred in 38 of 60 phakic eyes $(63 \%)$. Four preoperative factors were associated with final visual acuity of 20/60 or better: (1) initial vision of 20/100 or better, (2) shorter preoperative duration of blurred vision, (3) thin epiretinal membrane, and (4) absence of traction retinal detachment.

Localised epiretinal membranes may occur in the posterior pole of eyes without any associated retinal disease. Membranes may rarely be congenital or developmental, but more often they are acquired. Idiopathic epiretinal membranes can cause reduced vision and metamorphopsia by several mechanisms, including tissue covering and/or distorting the macula; low traction retinal detachment of the posterior pole; vascular leakage with intraretinal oedema; and obstruction of axoplasmic flow.'

Vitreous surgery is now used to remove the epiretinal tissue in selected cases. This paper reviews our experience treating 70 consecutive cases of idiopathic epiretinal membranes causing macular pucker and identifies prognostic factors for good postoperative visual acuity.

\section{Material and methods}

We retrospectively reviewed the records of 70 consecutive cases of idiopathic epiretinal membranes treated by vitrectomy and removal of epiretinal tissue from the macula. The term 'idiopathic' referred to epiretinal membranes occurring in the absence of any known vitreoretinal disease or pathology except for posterior vitreous detachment; therefore eyes with

Correspondence to Serge de Bustros, MD, Maumenee 115, 600 North Wolfe Street, Baltimore, MD 21205, USA. peripheral retinal tears were excluded. Patients under 40 years old were also excluded because such cases are sometimes considered to be congenital or developmental, though we believe they are usually acquired.

There were 34 males and 36 females. The patients' ages ranged from 42 to 85 years (median 68 years, mean 67 years). The right eye was involved in 37 cases and the left in 33 . The preoperative vision ranged from $20 / 50$ to hand motion at $1.5 \mathrm{~m}$ ( 5 feet). Five eyes were aphakic before vitrectomy and five other eyes had an intraocular lens. The follow-up interval ranged from 6 months to 65 months (median 12 months, mean 14 months). The vitrectomy techniques used to treat these cases have been previously published. ${ }^{1,-5}$

For each case 49 factors related to the preoperative examination, 38 factors relating to the operative procedure, and 23 factors related to the postoperative examination were tabulated. Items recorded for the history included age, sex, duration of blurred vision, duration of metamorphopsia, and types of previous ocular surgery. Findings from the preoperative examination included right or left eye, visual acuity, type of any lens opacity, aphakia or pseudophakia, intraocular pressure, vitreous opacity, posterior vitreous separation, type of epiretinal membrane (thin or more substantial), pigmentation 


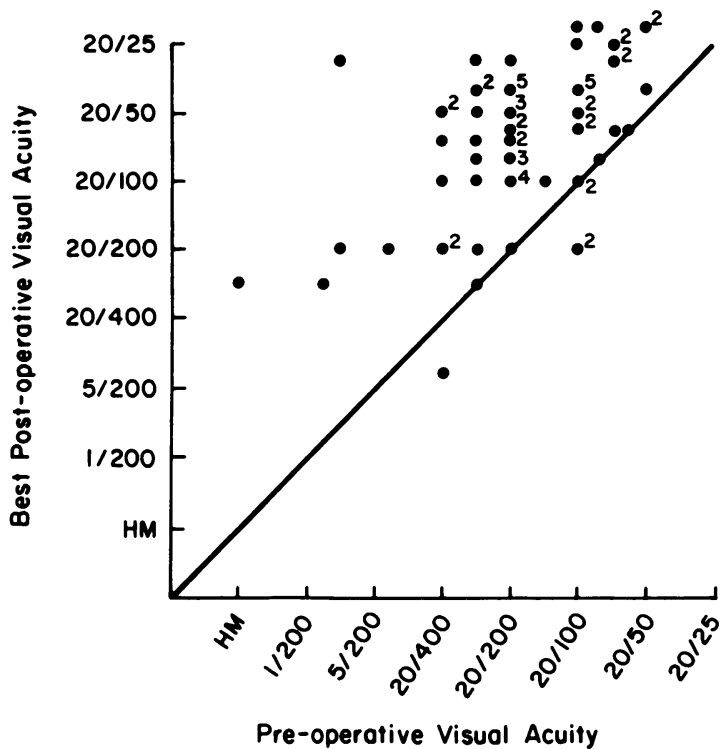

Fig. 1 Scattergram showing the preoperative visual acuity plotted against the best postoperative visual acuity. $H M=$ hand motion. Numbers adjacent to dots represent the number of eves present at a specific location. Absent numbers imply a single eve at that particular location on the graph. Dots above and to the left of the diagonal represent eyes with improved vision, dots below and to the right of the diagonal represent eyes with worse postoperative vision, and dots on the diagonal represent eyes with unchanged vision. Most eyes had improved vision after surgery.

of the epiretinal membrane, elevation of an edge of the epiretinal membrane, retinal vascular distortion, retinal striae, retinal dragging, presence of traction retinal detachment, macular thickening and/or cystoid macular oedema visible by slit lamp biomicroscopy, and epiretinal membrane in the fellow eye.

A statistical analysis was performed to determine which of the preoperative and intraoperative factors were associated with final visual acuity of $20 / 60$ or better. All comparisons of proportions were done by $\chi^{2}$ tests. The Mann-Whitney $U$ test was used to compare duration of symptoms between groups.

\section{Results}

The epiretinal membrane was removed from the fovea at the time of vitrectomy in all cases. However, in 28 eyes $(40 \%)$ remnants of the epiretinal membrane were left posterior to the equator. In three eyes (4\%) fragments of the epiretinal membranes were left in the macula. Mild petechial haemorrhages occurred at the time of surgery in 16 eyes $(23 \%)$.

Postoperatively the vision improved in 61 eyes $(87 \%)$ and remained the same in six eyes ( $9 \%$ ) (Fig. $1)$. Three eyes had worse vision after surgery. At

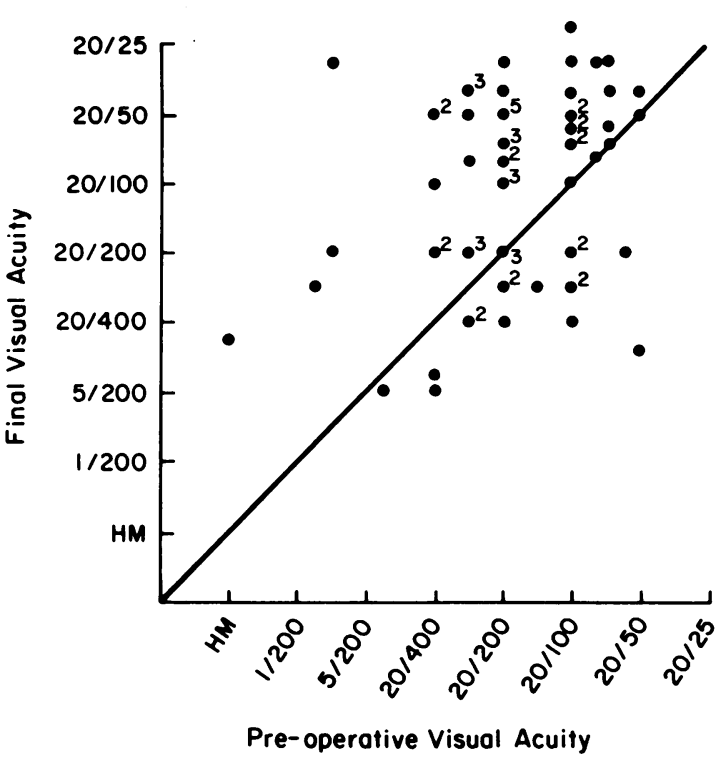

Fig. 2 Scattergram showing the preoperative visual acuity plotted against the final visual acuity. With longer follow-up fewer patients had improved vision after surgery, and more patients had worse vision. (Compare with Fig. I, and see Fig. 3 for more details about subsequent visual loss).

the time of final examination the vision was improved in 47 eyes $(67 \%)$, worse in 16 eyes $(23 \%)$, and unchanged in seven eyes (10\%) (Figs. 2 and 3$)$. The primary reason for subsequent decrease in visual acuity was the occurrence or progression of nuclear sclerosis of the crystalline lens. This occurred in 38 $(63 \%)$ of the 60 phakic eyes. Of the 14 eyes with decreased final visual acuity $11(79 \%)$ had development or progression of nuclear sclerosis. The median time for best postoperative vision was six months, whereas the median time for final vision was 12 months. There was no difference in the length of

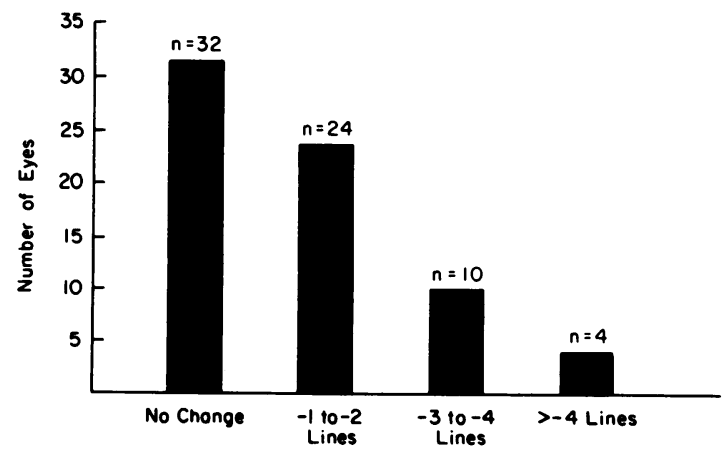

Fig. 3 Difference between best postoperative and final postoperative visual acuity in number of lines. The most common reason for subsequent visual loss is the occurrence or progression of nuclear sclerotic cataracts. 
follow-up between those patients whose vision improved and those that worsened.

Four preoperative factors were associated with a final visual acuity of $20 / 60$ or better: initial visual acuity of $20 / 100$ or better, shorter duration of blurred vision, thin epiretinal membrane, and absence of traction retinal detachment (Table 1).

A complete posterior vitreous detachment, as determined from the preoperative examination and/or intraoperative findings, was present in 55 eyes $(79 \%)$. Seven eyes $(10 \%)$ had no posterior vitreous detachment. The remaining eight eyes had a partial posterior vitreous detachment. In six eyes the posterior vitreous was partially attached to the epiretinal membrane.

The epiretinal membrane had a thin, cellophanelike appearance in 39 eyes $(56 \%)$, and it was thicker in 31 eyes $(44 \%)$. In 43 eyes $(61 \%)$ a partly raised edge was visible preoperatively. Twelve eyes $(17 \%)$ had various degrees of cystoid macular oedema visible preoperatively by contact lens examination. Fluorescein angiography was not routinely performed. Eleven eyes $(16 \%)$ had an epiretinal membrane of less severity involving the macula in the fellow eye.

\section{COMPLICATIONS}

Peripheral retinal tears were identified at the end of the operation in four eyes $(6 \%)$. These were treated by cryotherapy, partial fluid/air exchange, and postoperative face-down positioning. One eye developed a peripheral retinal detachment postoperatively, and it was successfully treated with a scleral buckling procedure. No posterior retinal breaks occurred during removal of the epiretinal membranes.

Angle closure glaucoma occurred postoperatively in one eye. It was treated with a laser iridotomy. Progressive nuclear sclerotic lens changes developed in $38(63 \%)$ of 60 phakic eyes. The causes of this complication are unknown, and it is being investigated further. At the time of final examination recurrent epiretinal membranes affecting the macula were found in three eyes $(4 \%)$. There was no apparent reason for the recurrence of epiretinal membranes in these three eyes.

\section{Discussion}

Idiopathic epiretinal membranes causing macular pucker and impaired vision are a well known acquired clinical condition. Vitreous surgery is now used to remove the epiretinal membrane from the macular area in selected cases if there is substantial visual loss. However, visual recovery is usually incomplete. There are no firm criteria for recommending surgery, and the indications may vary
Table 1 Vitrectomy for idiopathic macular pucker: prognostic factors for final visual acuity

\begin{tabular}{lrr}
\hline Preop. factor & $\begin{array}{l}\text { Final postoperative vision- } \\
\text { number of eyes }(\%)\end{array}$ \\
\cline { 2 - 3 } & $\geqslant 20 / 60$ & $<20 / 60$ (p value) \\
\hline Preop. VA $\geq 20 / 100$ & $21(81)$ & $5(19)$ \\
Preop. VA $<20 / 100$ & $18(41)$ & $26(59)$ \\
Thin & $26(67)$ & $13(33)$ \\
More substantial & $12(40)$ & $18(60)$ \\
TRD & $2(18)$ & $9(82)$ \\
No TRD & $37(63)$ & $22(27)$ \\
Duration of blurred vision (median) & 350 days & 747 days $(<0.007)^{*}$ \\
& &
\end{tabular}

$\mathrm{VA}=$ visual acuity.

TRD = traction retinal detachment.

${ }^{*} \mathrm{p}$ Value based on $\chi^{2}$ test.

tp Value based on Mann-Whitney $U$ test.

depending on the visual needs of each patient. However, most of the cases $(84 \%)$ in our series had preoperative visual acuity of $20 / 100$ or worse in the involved eye.

Idiopathic epiretinal membranes present a unique opportunity to study the effects of epiretinal tissue on visual function, because these eyes do not have other prior or concurrent retinal disease. Identification of prognostic indicators of visual results helps in case selection for surgery and may aid in understanding the pathogenesis of visual loss in this condition. In our series four factors were associated with final visual acuity of $20 / 60$ or better: (1) initial visual acuity of $20 / 100$ or better, (2) shorter duration of blurred vision, (3) thin, cellophane-like appearance of the epiretinal membrane, and (4) absence of posterior traction retinal detachment.

Relatively good initial visual acuity and shorter duration of blurred vision have also been found to be prognostic indicators of good postoperative vision after vitrectomy for other types of epiretinal membranes causing macular pucker. This probably indicates less initial macular damage. ${ }^{6}$ Epiretinal membranes with a thin (cellophane) appearance also had a better visual prognosis. This agrees with the observations of others, though the reason for this association is not certain. ${ }^{\text {? }}$

In 11 cases the traction caused by the epiretinal tissue produced a limited low retinal detachment. This correlated with a poorer visual result, probably because of further disturbance of the retinal architecture and interference with the retinal pigment epithelium-photoreceptor complex.

Peripheral retinal tears were found at the end of surgery in four eyes $(6 \%)$. These were successfully treated by cryotherapy and partial fluid/air exchange. This underscores the need for careful examination of the peripheral retina by indirect ophthalmoscopy with scleral depression at the end of the operation. 
The high incidence of progressive postoperative nuclear sclerosis found in this series is of obscure aetiology. Michels had previously reported an incidence of $34 \%$ in 90 cases. ${ }^{8}$ Many reasons could explain the higher incidence of postoperative nuclear sclerosis in this series, including longer follow-up and selection of homogeneous cases of epiretinal membranes as compared with the previous series of mixed aetiology. Margherio et al. ${ }^{9}$ attribute their lower incidence of postoperative nuclear sclerosis $(12.5 \%)$ to a variety of factors, including shorter operating time, distance of infusion port from the lens, type of irrigating fluid, and different surgical techniques, leaving anterior vitreous behind the lens. Recently, however, McDonald et al. ${ }^{10}$ using similar surgical techniques, observed this complication in $39 \%$ of their cases. Clearly the postoperative occurrence of nuclear sclerosis deserves further study. It is being investigated further.

The authors thank Cheryl Enger, MS, from the Wilmer Biostatistical Center, for statistical analysis, and Carol Srnec for assistance in the preparation of the manuscript.

JTT was supported in part by a Heed and Heed-Knapp Ophthalmic Foundation Fellowship 1984-5. Yale University Department of Ophthalmology and Visual Science, New Haven.

\section{References}

1 Michels RG. Vitreous surgery for macular pucker. Am J Ophthalmol 1981: 92: 628-39.

2 Barr CC, Michels RG. Idiopathic nonvascularized epiretinal membranes in young patients: report of six cases. $A n n$ Ophthalmol 1982; 14: 335-41.

3 Machemer R. Die chirurgische Entfernung von epiretinalen Makula-Membranen (macular puckers). Klin Monastbl Augenheilkd 1978; 173: 36-42.

4 Charles S. Vitreous microsurgery. Baltimore: Williams and Wilkins, 1981: 131-3.

5 Michels RG. Vitreous surgery. St Louis: Mosby, 1981: 357-62.

6 Rice TA, de Bustros S, Michels RG, Thompson JT, Debannc SM, Rowland DY. Prognostic factors in vitrectomy for epiretinal membranes of the macula. Ophthalmology 1986; 93: 602-10.

7 Trese MT, Chandler DB, Machemer R. Macular pucker. I. Prognostic criteria. Graefes Arch Clin Exp Ophthalmol 1983: 221: $12-5$.

8 Michels RG. Vitrectomy for macular pucker. Ophthalmology 1984; 91: 1384-8.

9 Margherio RR, Cox MS Jr, Trese MT, Murphy PL, Johnson J, Minor LA. Removal of epimacular membranes. Ophthalmology 1985; 92: 1075-83.

10 McDonald HR, Verre WP, Aaberg TM. Surgical management of idiopathic epiretinal membranes. Ophthalmology 1986; 93: 978-83.

Accepted for publication 21 June 1987. 